\title{
Sample selection and validity of exposure-disease association estimates in cohort studies
}

Corresponding author:

Costanza Pizzi

Via Santena 7, 10126 Torino, Italy

costanza.pizzi@1shtm.ac.uk

Tel: +390116334628

Fax: +390116334664

Author:

Costanza Pizzi ${ }^{1,2}$, Bianca De Stavola ${ }^{2}$, Franco Merletti ${ }^{1}$, Rino Bellocco ${ }^{3,4}$, Isabel dos Santos Silva $^{2}$, Neil Pearce ${ }^{5}$, Lorenzo Richiardi ${ }^{1}$

Author Affiliation:

${ }^{1}$ Cancer Epidemiology Unit, CeRMS and CPO-Piemonte, University of Turin, Italy

2 Department of Epidemiology and Population Health, London School of Hygiene and Tropical Medicine, London, UK.

${ }^{3}$ Department of Statistics, University of Milano Bicocca, Milan, Italy

${ }^{4}$ Department of Medical Epidemiology and Biostatistics, Karolinska Institutet, Stockholm, Sweden

${ }^{5}$ Centre for Public Health Research, Massey University Wellington Campus, New Zealand.

Keywords: Directed Acyclical Graphs; Selection bias; Confounding; Monte Carlo Simulations

Word Count: Text: 3,277; Abstract: 240; References: 24; Figures: 2; Tables: 1; Supplementary Tables: 1 . 


\begin{abstract}
BACKGROUND: Participants in cohort studies are frequently selected from restricted source populations. It has been recognised that such restriction may affect the study validity.

METHODS: We assessed the bias that may arise when analyses involve data from cohorts based on restricted source populations, surprisingly an area little studied in quantitative terms. We used Monte Carlo simulations based on a setting where the exposure and one risk factor for the outcome, which are not associated in the general population, influence selection into the cohort. All the parameters involved in the simulations (i.e. the prevalence and effects of exposure and risk factor on both the selection and outcome process, the selection prevalence, the baseline outcome incidence rate, and the sample size) were allowed to vary to reflect real life settings.

RESULTS: The simulations show that when the exposure and risk factor are strongly associated with selection (odds ratios of 4 or 0.25 ) and the unmeasured risk factor is associated with a disease hazard ratio of 4 , the bias in the estimated log odds ratio for the exposure-disease association is \pm 0.15 . When these associations decrease to values more commonly seen in epidemiological studies (e.g., odds and hazard ratios of 2 or 0.5 ), the bias in the log odds ratio drops to just \pm 0.02 .
\end{abstract}

CONCLUSIONS: Using a restricted source population for a cohort study will, under a range of sensible scenarios, produce only relatively weak bias in estimates of the exposure-disease associations.

\title{
Introduction
}

Selection of study subjects from restricted source populations according to pre-specified criteria is an approach that is frequently used in cohort studies. The purposes of such restrictions are to enhance study feasibility and to increase the prevalence of exposure or the completeness of follow-up, thereby increasing study validity and precision. Typically this may involve recruiting participants from a subgroup of the general population, rather than sampling directly from the entire general population. Such subgroups may be defined on the basis of occupation, gender, geographical area, birth cohort, etc. The British Doctors Study [1] and the Nurses' Health Study,[2] occupational cohorts,[3] follow-up of participants in specific events,[4] analyses restricted to specific subgroups of the population, such as non-smokers,[5] ancillary analyses of non-randomized exposures in randomized studies,[6] follow-up studies of screening attendants [7] are all examples of cohort studies based on restricted samples.

Undoubtedly, restriction of the source population may introduce problems of generalizability of the study findings, but this also applies to studies that are based in the general population (e.g., most cardiovascular epidemiology involves cohort studies in specific communities rather than true general population samples). We will therefore not consider issues of generalizability here; rather, our focus is on whether using a restricted source population may affect the validity of the exposure-disease associations. $[8,9]$ In particular, bias will be introduced if a risk factor for disease is not associated with exposure in the general population but is associated with exposure in the study population, as a result of the selection process. Such biases can be represented using Directed Acyclical Graphs (DAGs).[8, 10-13] The example depicted in 
Figure 1a, represents a population in which there is no association between an exposure (E) and a disease (D); there is another risk factor $(\mathrm{R})$ for the disease, but this is not a source of confounding as it is not associated with the exposure. However, E and R both affect the likelihood of being selected $(S=1)$ into the study. When analyses are restricted to the selected subjects, there is an inherent conditioning on $S$ (as represented by a square around $S$ in Figure 1b), which leads to a spurious association between $\mathrm{E}$ and $\mathrm{R}$ (represented by a dashed line). Under this scenario, even if $\mathrm{E}$ has no causal effect on $\mathrm{D}$, the backdoor path $\mathrm{E}-\mathrm{R}-\mathrm{D}$ is opened and the estimated associational relative risk between the exposure and the disease $\left(A R R_{D E}\right)$ may differ from the causal relative risk $\left(\mathrm{CRR}_{\mathrm{DE}}\right)$. This could, for example, be the situation in a cohort study of the effect of obesity (E) on breast cancer (D) based on breast cancer screening participants (the restricted source population). In this example, obese women (E) are less likely to attend the screening programs [14] while women with a family history of breast cancer (R) are more likely to participate. Among those who attend screening (i.e. conditioning on those with $S=1$ ), obesity (E) and family history of breast cancer (R) become positively correlated. In fact an obese woman is more likely to have a family history of breast cancer within the selected sample than in the general population, because otherwise she may not participated in the screening program. As a result family, history of breast cancer is a confounder of the obesity-breast cancer association if studied among screening attendees but not - or to a less extent - in the general population.

This type of bias has been extensively discussed in the causal inference literature from a theoretical point of view.[8, 9] Hernan and colleagues' 2004 paper on selection bias provides the conceptual framework and indicates that if the risk factor associated with the selection process is known and measured it is possible to adjust for selection bias, whereas if the risk factor is unmeasured, the effect estimates may be biased. However, although the theoretical basis of selection bias is clear, there have been few attempts to quantify the likely strength of such biases. One exception is that of Greenland,[15] who studied the setting of Figure 1b with dichotomous exposure and outcome variables, employing methods originally developed to quantify the impact of unmeasured confounding.[16] He calculated the likely maximum strength of the bias in the estimation of the E-D association in the $S=1$ stratum as a function of the odds ratios (ORs) corresponding to the true associations depicted in Figure $1 \mathrm{~b}$ (i.e. $\mathrm{OR}_{\mathrm{SE}}, \mathrm{OR}_{\mathrm{SR}}, \mathrm{OR}_{\mathrm{DR}}$ ). However, it is not clear how these results apply to cohort studies. Because of the increasing frequency of cohort studies based on selected populations, such as the internet-based birth cohort studies based in Italy (NINFEA cohort) and New-Zealand (ELFS),[17] quantifying the potential biases involved in analysing such data is timely and relevant.

Our aim is therefore to study the extent of these biases. We use simulations to mimic a variety of cohort restrictions and disease settings and examine the consequent bias in the estimated exposure hazard (or rate) ratio (HR) of disease. We then discuss these results in terms of whether, and under what circumstances, the resulting selection bias is serious enough to strongly bias the exposure effect estimates. For simplicity, we will assume throughout the paper that there is negligible random variation, that all variables are measured without error, and that there is uninformative censoring.

\section{Sample selection and disease risk factors}

As previously recognised, $[9,18]$ a fundamental characteristic of selection bias in restricted cohort studies is that the selection process makes a disease risk factor, which may not be associated with the exposure in the general population, become associated with the exposure among the study population and therefore act as a confounder. 
Confounders in the general population and risk factors that become confounders in a restricted source population are usually indistinguishable when the study is analysed. Although typically some disease risk factors (i.e. potential confounders) are known a priori, it is seldom known whether these are associated with the exposure of interest in the specific population in which the study will be carried out. Both in general population-based and restricted cohorts, therefore, researchers attempt to collect information on all known and suspected important risk factors of the disease in the population that they are studying, regardless of their expectations about whether these are associated with the exposure or not. The example of the association between smoking and socioeconomic position (SEP) illustrates well this point. Depending on the population and the calendar period, SEP can be positively or negatively associated, or not associated at all, with smoking. Researchers aiming to estimate the association between smoking and mortality will always attempt to collect information on SEP and, in most instances, will control for it, irrespective of whether the confounding effect of SEP is due to a real association between SEP and smoking in the general population or a spurious association caused by the sample selection process.

Another possible consequence of the selection mechanism is a change in magnitude, and in extreme cases direction, of the confounding effect of a risk factor. This may occur if the strength of the association between the risk factor and the exposure in the selected sample differs from that originally present in the general population. For example, when two (parent) variables influence a third (child) variable in the same direction, conditioning on the child variable likely leads to a negative association between the parent variables.[8] Thus, if an exposure and a confounder influence the selection process in the same direction, the original association between exposure and confounder will be reduced in the subset of those who participate if they were originally positively associated, or increased if their original association was negative. For example, in many populations smoking and physical exercise are negatively associated. In a hypothetical study restricted to blood donors, who typically have a healthy lifestyle and thus smoke less and exercise more than the average individual in the general population, the sample selection would add a positive association between smoking and physical exercise. Therefore, the original negative association present in the general population would be, if anything, attenuated among blood donors.

In the next section, we use simulations to quantify the likely extent of selection bias arising from the use of restricted cohorts.

\section{Quantification of the bias}

\section{Methods}

We conducted Monte Carlo simulations of alternative settings corresponding to the scenario of Figure $1 b$ to quantify the resulting bias in the estimation of the E-D effect when conditioning on $S=1$ and not adjusting for R.[19] The generation process of the four variables of Figure $1 \mathrm{~b}$ is described below.

We generated $\mathrm{E}$ and $\mathrm{R}$ as marginally independent binary variables, with prevalence, respectively $\mathrm{P}_{E}$ and $\mathrm{P}_{R}$, initially set equal to 0.5 in the source population. They were later allowed to decrease to 0.25 for $\mathrm{P}_{\mathrm{E}}$ and to 0.1 for $\mathrm{P}_{\mathrm{R}}$, in order to investigate scenarios more frequently addressed by epidemiologists. 
The binary variable $\mathrm{S}$ was generated using a logistic regression model with baseline prevalence, $\mathrm{P}_{\mathrm{S}}$, equal to 0.5 and with the $\mathrm{ORs}$ for the explanatory binary variables $\mathrm{E}$ and $\mathrm{R}$ taking values $0.25,0.33,0.50,2,3$ and 4 . Specifically, with ?s indicating the $\log$ (odds) of $S=1$ among the

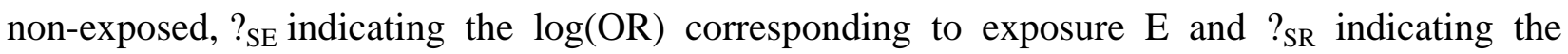
$\log (\mathrm{OR})$ corresponding to $\mathrm{R}$, the generating model was:

$$
\operatorname{logit}(S=1)=?_{S}+?_{S E} E+?{ }_{S R} R
$$

A more complex model that included an interaction term between $\mathrm{E}$ and $\mathrm{R}$ was also considered:

$$
\operatorname{logit}(S=1)=?_{S}+?_{S E} E+?_{S R} R+?_{\text {inter }} E^{*} R
$$

with $\mathrm{OR}_{\text {inter }}$, corresponding to $\exp \left(\right.$ ? $\left._{\text {inter }}\right)$, set at values 0.5 or 2 . The interaction term was introduced to examine more realistic selection settings. For example, in the first empirical demonstration of the Berkson's bias, Roberts and colleagues found that not only chronic conditions increase the chance of hospitalization, but often they also interact more than multiplicatively.[20]

We generated time to the outcome $\mathrm{D}$ assuming a constant rate ?, i.e. we assumed that time to event followed an exponential distribution.[21] The baseline rate $?_{0}$ was set equal to $0.01,0.03$, or 0.06 events/year, with administrative censoring time set at 5 years. The rate ? was allowed to be affected only by $\mathrm{R}$, with hazard ratios $\mathrm{HR}_{\mathrm{DR}}$ taking values $0.25,0.33,0.50,2,3$ and 4 , while we

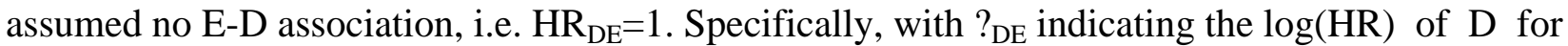
the exposure $\mathrm{E}$ and $?_{\mathrm{DR}}$ indicating the $\log (\mathrm{HR})$ of $\mathrm{D}$ for the risk factor $\mathrm{R}$, the $\log$ rate function for $\mathrm{D}, \log (?)$, was defined as:

$$
\log (?)=\log ?_{0}+?_{D E} E+?_{D R} R
$$

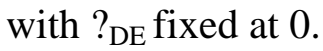

We generated a total of 1,000 Monte Carlo simulated datasets of 5,000 subjects for each combination of the parameters described above. We also used a size of 2,500 subjects, increasing the number of simulations $(n=2,000)$, to deal with the greater impact of random variation.

In each simulated dataset, we estimated two main parameters in the stratum $S=1$ (which sample size varies as a consequence of the selected parameters for the selection process): the association between $\mathrm{E}$ and $\mathrm{R}\left(\mathrm{OR}_{\mathrm{ER}}\right)$ and the association between $\mathrm{E}$ and $\mathrm{D}\left(\mathrm{HR}_{\mathrm{DE}}\right)$ which is induced by the selection process. The estimate of $\mathrm{HR}_{\mathrm{DE}}$ was obtained fitting a Cox proportional hazards regression model with no adjustment for R.[22] We then calculated the bias in the E-D association as the difference between zero, i.e. the true value of ? $_{\mathrm{DE}}$, and the logarithm of the estimated $\mathrm{HR}_{\mathrm{DE}}$. For each scenario, we summarised the bias, and the estimated values of ? ${ }_{\mathrm{DE}}$, in terms of means, standard deviation, and $5^{\text {th }}$ and $95^{\text {th }}$ percentiles.

\section{Results}

We first considered the situation with prevalence of $\mathrm{E}$ and $\mathrm{R}$ both equal to $0.5, \mathrm{OR}_{\text {inter }}=1$ (i.e. no multiplicative interaction), and $?_{0}=0.03$ (the "reference scenario" in Table 1). As expected, the size of the bias in the estimation of $\mathrm{OR}_{\mathrm{DE}}$ depended on: $\mathrm{i}$ ) the induced association between the exposure and the risk factor $\left(\mathrm{OR}_{\mathrm{ER}}\right)$, which increased in absolute terms with the absolute size of $\mathrm{OR}_{\mathrm{SR}}$ and $\mathrm{OR}_{\mathrm{SE}}$; and ii) the magnitude of the association between the risk factor and the disease $\left(\mathrm{HR}_{\mathrm{DR}}\right)$. The largest values of the bias in the $\log$ odds ratio were \pm 0.15 (Table 1 , "reference scenario"), which were reached when $\mathrm{OR}_{\mathrm{SE}}, \mathrm{OR}_{\mathrm{SR}}$ and $\mathrm{HR}_{\mathrm{DR}}$ were furthest from the null value (i.e. equal to 0.25 or 4$)$. Note that in Table 1 the range for $\log \left(\mathrm{OR}_{\mathrm{ER} \mid \mathrm{S}=1}\right)$ is not symmetrical because the magnitude of the association induced by the selection between $\mathrm{E}$ and $\mathrm{R}$ also depends on the prevalence of $\mathrm{S}$ in the population $\left(\mathrm{P}_{\mathrm{s}}\right)$, with the strongest association obtained when $\mathrm{P}_{\mathrm{S}}=0.5$. The complete results for all combinations of the values of $\mathrm{OR}_{\mathrm{SE}}, \mathrm{OR}_{\mathrm{SR}}$ and $\mathrm{HR}_{\mathrm{DR}}$ are reported in Supplementary Table 1 . The mean bias decreased from \pm 0.15 to just \pm 0.02 when the three 
ORs/HRs were equal to 2 or 0.5 .

When an interaction term between $\mathrm{E}$ and $\mathrm{R}$ was included in the model generating $\mathrm{S}$, the induced E-R association increased considerably (Figure 2), up to a $\log (\mathrm{OR})$ of -0.98 (Table 1, row 2) when $\mathrm{OR}_{\mathrm{SE}}$ and $\mathrm{OR}_{\mathrm{SR}}$ were equal to 0.25 and the $\mathrm{OR}_{\text {inter }}$ was 0.5 . The bias increased accordingly, ranging from -0.24 to 0.27 (Table 1, row 2). This situation is equivalent, in terms of induced bias, to those involving very strong marginal associations with selection. It is clear, from Figure 2, that the impact of the interaction is not the same for all the parameter combinations, as the magnitude of the induced E-R association is strengthened or reduced according to the sign of the interaction term but also to the size of the stratum of subjects exposed to both $\mathrm{E}$ and $\mathrm{R}$.

Neither the prevalence of the exposure E (Table 1 row 3) nor the baseline rate for the disease D (Table 1 rows 4-5), or the sample size (Table 1, row 6) affected the extent of the bias. Conversely, the prevalence of $\mathrm{R}$, which becomes a confounder of the E-D association when $\mathrm{S}=1$, had a non-marginal effect. For a given value of the induced E-R association, the bias reached its peak when the prevalence of $R$ among the selected subjects ( $S=1$ stratum) was 0.5 . For this reason when the population prevalence of $\mathrm{R}$ was set equal to 0.1 instead of 0.5 , the range of the mean bias decreased to $(-0.12 ; 0.07)$ (Table 1 , row 7$)$.

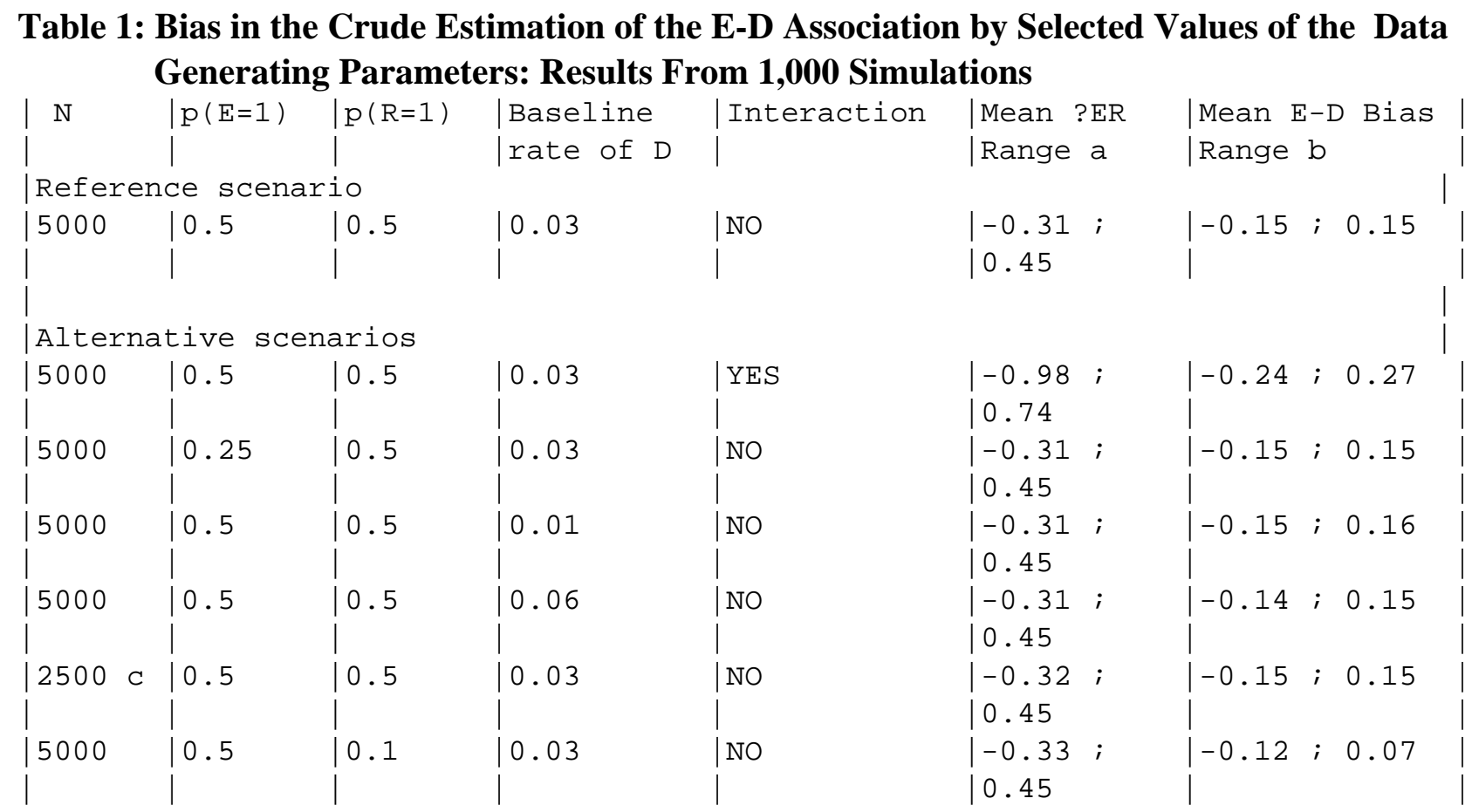

E, exposure; R, risk factor; $\mathrm{D}$, disease

a ?ER expressed as $\log (\mathrm{OR})$

b ? $\mathrm{DE}$ expressed as $\log (\mathrm{HR})$

c Results from 2,000 simulations

\section{Discussion}

Conducting cohort studies in a restricted sample of the general population may offer several advantages, including more precise measurement of the exposure, higher exposure prevalence, enhanced feasibility of the study, better control of confounding, increased sample size, higher recruitment rates, and a higher completeness of follow-up. These advantages should be balanced against issues of validity. 
In this paper we have shown, via simulations, that the possible bias introduced by restriction of the source population, is usually weak when internal comparisons are carried out within the cohort, with a maximum bias in the $\log (\mathrm{HR})$ of \pm 0.15 .

These results are in agreement with those of Greenland,[15] who used an analytical approach to quantify the maximum selection bias in settings where the outcome risk is rare so that the analysis of cohort data can be performed using logistic regression. Our simulations add further insight to these results as we examined a wide range of disease and selection parameters, including exposure and risk factor prevalence, which highlighted their individual role in influencing the extent of the bias. Further, we considered settings where exposure and risk factor interact when influencing the selection process. Some additional points are warranted.

First, the bias is necessarily small when the association between the exposure of interest and the selection process is relatively weak (i.e. $0.5<\mathrm{OR}<2$ ). In particular when the exposureselection OR is equal to 2 or 0.5 , while the risk factor-selection OR and the risk factor-disease HR are allowed to take values up to 4 or down to 0.25 , the maximum bias in the estimated exposuredisease association is within the \pm 0.07 range (on the log hazard scale). For example consider the Million Women Study, a cohort nested within the breast screening programme in the UK.[7] From the study carried out to compare the characteristics of the study participants with the rest of the population (women who attended the screening but did not join the study plus not attendants),[23] the participation OR for current use of hormone replacement therapy (HRT), which is the main exposure of interest of the study, was derived. This estimated OR was about 1.6. On the basis of this information it is possible to assume that, in this cohort, the bias introduced by the baseline selection on the estimates of the HRT's effect on the outcome of interest would be negligible.

Second, selection must be associated with one or more unmeasured or unknown disease risk factors in order to introduce bias. However, unknown or unmeasured disease risk factors can introduce bias whether or not the cohort is based on the general population or a restricted source population; in the latter case, the sample selection can either increase or decrease the overall bias with a magnitude and direction difficult to predict if there are multiple risk factors involved.[24]

Third, we have shown that even when all of the associations involved in the selection and outcome mechanisms are reasonably large (e.g., all ORs/HRs of 4.0 or 0.25 ), the prevalence of the risk factor $\mathrm{R}$ is about $50 \%$ and there is no adjustment for $\mathrm{R}$, the resulting bias is relatively weak (i.e. \pm 0.15 on the $\log$ scale). This is reassuring, as this scenario is rather extreme and very unlikely to occur in practice. Besides, a disease risk factor with a $50 \%$ prevalence and a disease hazard ratio of 4.0 would have an attributable fraction of $60 \%$ and is therefore unlikely not to have been known and measured when a study is planned.

The scenarios considered in our simulations were restricted to binary exposure and binary risk factor and assumed no association between the exposure and the risk factor in the general population. A limitation is that we examined only the case of a single unmeasured determinant of the disease that also influences the selection process. However we believe it is unlikely that multiple and independent important disease risk factors would affect the sample selection. It is indeed reasonable to consider $\mathrm{R}$ as a vector resulting from the combination of a set of correlated risk factors, all moderately associated with S. Finally, we only showed the findings derived from the analyses based on the assumption of a null causal association between the exposure and the outcome of interest; however choosing a true associational value, ? ${ }_{\mathrm{DE}}$, different from zero would not modify the simulation results and therefore our conclusions. 
We conclude that using a restricted source population for a cohort study will, under a range of sensible scenarios, produce only weak bias in estimates of the exposure-disease associations. On the other hand, the use of such restrictions may increase the response rate and the exposure prevalence, as well as being the only feasible approach in many circumstances.

Competing Interest: None declared.

\section{Funding}

The study was conducted within projects partially funded by Compagnia SanPaolo/FIRMS, the Piedmont Region, the Italian Ministry of University and Research (MIUR), the Italian Association for Research on Cancer (AIRC) and the Massey University Research Fund (MURF). The Centre for Public Health Research is supported by a Programme Grant from the Health Research Council of New Zealand.

\section{Copyright licence statement}

"The Corresponding Author has the right to grant on behalf of all authors and does grant on behalf of all authors, an exclusive licence (or non exclusive for government employees) on a worldwide basis to the BMJ Publishing Group Ltd and its licencees, to permit this article (if accepted) to be published in JECH and any other BMJ Group products and to exploit all subsidiary rights, as set out in our licence (http://jech.bmjjournals.com//ifora/licence.pdf)"

\section{References}

1 Doll R, Peto R, Boreham J, et al. Mortality in relation to smoking: 50 years' observations on male British doctors. BMJ 2004;328:1519.

2 Colditz GA, Hankinson SE. The Nurses' Health Study: lifestyle and health among women. Nat Rev Cancer 2005;5:388-96.

3 Magnani C, Ferrante D, Barone-Adesi F, et al. Cancer risk after cessation of asbestos exposure: a cohort study of Italian asbestos cement workers. Occup Environ Med 2008;65:164-70. 4 Lagerros YT, Bellocco R, Adami HO, et al. Measures of physical activity and their correlates: the Swedish National March Cohort. Eur J Epidemiol 2009;24:161-9.

5 Vineis P, Airoldi L, Veglia F, et al. Environmental tobacco smoke and risk of respiratory cancer and chronic obstructive pulmonary disease in former smokers and never smokers in the EPIC prospective study. BMJ 2005;330:277.

6 Beattie MS, Costantino JP, Cummings SR, et al. Endogenous sex hormones, breast cancer risk, and tamoxifen response: an ancillary study in the NSABP Breast Cancer Prevention Trial (P1). J Natl Cancer Inst 2006;98:110-5.

7 The Million Women Study: design and characteristics of the study population. The Million Women Study Collaborative Group. Breast Cancer Res 1999;1:73-80.

8 Glymour MM. Using causal diagrams to understand common problems in social epidemiology. In: Jossey-Bass, ed. Methods in social epidemiology. San Francisco 2006.

9 Hernan MA, Hernandez-Diaz S, Robins JM. A structural approach to selection bias. Epidemiology 2004;15:615-25.

10 Greenland S, Pearl J, Robins JM. Causal diagrams for epidemiologic research. Epidemiology 1999;10:37-48.

11 Hernan MA, Hernandez-Diaz S, Werler MM, et al. Causal knowledge as a prerequisite for confounding evaluation: an application to birth defects epidemiology. $A m \quad J$ 
Epidemiol 2002;155:176-84.

12 Pearl J. Causal diagrams for empirical research. Biometrika 1995;82:669-88.

13 Pearl J. Causality: Models, Reasoning, and Inference. Cambridge, UK: Cambridge University Press 2000.

14 Ferrante JM, Chen PH, Crabtree BF, et al. Cancer screening in women: body mass index and adherence to physician recommendations. Am J Prev Med 2007;32:525-31.

15 Greenland S. Quantifying biases in causal models: classical confounding vs colliderstratification bias. Epidemiology 2003;14:300-6.

16 Yanagawa T. Case-Control Studies: Assessing the Effect of a Confounding Factor. Biometrika 1984;71:191-4.

17 Richiardi L, Baussano I, Vizzini L, et al. Feasibility of recruiting a birth cohort through the Internet: the experience of the NINFEA cohort. Eur J Epidemiol 2007;22:831-7.

18 Rothman KJ, Greenland S, Lash TL. Distinguishing Selection Bias from Confounding. Modern Epidemiology. Philadelphia: Lippincott Williams \& Wilkins 2008:136-7.

19 Burton A, Altman DG, Royston P, et al. The design of simulation studies in medical statistics. Stat Med 2006;25:4279-92.

20 Roberts RS, Spitzer WO, Delmore T, et al. An empirical demonstration of Berkson's bias. J Chronic Dis 1978;31:119-28.

21 Bender R, Augustin T, Blettner M. Generating survival times to simulate Cox proportional hazards models. Stat Med 2005;24:1713-23.

22 Clayton D, Hills M. Statistical Models in Epidemiology. Oxford Oxford University Press 1993.

23 Banks E, Beral V, Cameron R, et al. Comparison of various characteristics of women who do and do not attend for breast cancer screening. Breast Cancer Res 2002;4:R1.

24 VanderWeele TJ, Hernan MA, Robins JM. Causal directed acyclic graphs and the direction of unmeasured confounding bias. Epidemiology 2008;19:720-8.

\section{Figure legends}

Figure 1: Diagram of a cohort based on a selected sample. a) In the population the exposure of interest $(\mathrm{E})$ is not associated with the disease of interest (D) that is caused by a risk factor $(\mathrm{R})$. Both $\mathrm{E}$ and $\mathrm{R}$ affect the probability of being selected $(\mathrm{S})$ as a member of the cohort. b) The study is carried out in the selected sample and, therefore, there is an inherent conditioning on $\mathrm{S}$ (a box around a variable means conditioning for that 
variable) which generated an induced association between $\mathrm{E}$ and $\mathrm{R}$ (represented by a dashed line)

Figure 2: Mean OR of the Induced E-R Association in the Stratum of Those Selected $(S=1)$ by Selected Values of the Association of the Exposure $\left(\mathrm{OR}_{\mathrm{SE}}\right)$ and the Risk Factor $\left(\mathrm{OR}_{\mathrm{SR}}\right)$ With the Selection Process and of the E-R Interaction $\left(\mathrm{OR}_{\text {inter }}\right)$ : Results From 1,000 Simulations 